\title{
Secondary de-bulking surgery in recurrent ovarian cancer
}

\author{
Interview with Philipp Harter ${ }^{1}$ and Nicoletta Colombo ${ }^{2}$ \\ by Claudia Marchetti ${ }^{3}$ and Federica Tomao ${ }^{3}$
}

\author{
Surgery perspective: \\ Philipp Harter ${ }^{1}$ \\ Medical oncology perspective: \\ Nicoletta Colombo ${ }^{2}$
}

\section{Introduction}

The role of secondary cytoreductive surgery has been extensively debated, and the majority of data come from retrospective series. The only randomized trial (DESKTOP III) results on this topic were presented at the recent annual meeting of the American Society of Clinical Oncology (ASCO) [1].

The study was a phase III trial that tested the efficacy of secondary cytoreductive surgery in recurrent ovarian cancer with overall survival (OS) being the primary endpoint. Patients included into the study were affected by platinum-sensitive disease with a positive AGO (Arbeitsgemeinschaft Gynäkologische Onkologie) score (Eastern Cooperative Oncology Group [ECOG] performance status 0 , ascites $\leq 500 \mathrm{~mL}$ and complete resection at initial surgery). Women were randomly assigned to receive second-line chemotherapy alone or cytoreductive surgery followed by chemotherapy.

The study found that the surgical approach followed by chemotherapy resulted in a better progression-free survival (PFS) compared to chemotherapy administration alone (14.0 vs 19.6 months, $\mathrm{p}<0.001)$ with a longer median time to start of first subsequent treatment. However, the benefit was statistically significant only for those patients in whom a complete surgical removal of the disease had been obtained, emphasizing the prognostic role of residual tumor in secondary treatment, as in the frontline setting. This study also suggests that adequate patient selection is necessary in order to establish which patients might potentially benefit from the surgical approach.

Data on the primary endpoint, OS, are still too immature for a final analysis. However, the observed pooled 2-years survival rate was $83 \%$, which was much higher than the $55-66 \%$ estimated in the study protocol for the overall trial population.
In this interview, we asked for opinions about secondary de-bulking surgery in platinum-sensitive disease from two eminent experts in the field, Dr. Philipp Harter (principal investigator of the DESKTOP III trial) and Prof. Nicoletta Colombo (an expert on the medical treatment of gynecologic malignancies).

\section{Do you believe that secondary surgery is superior to second-line chemotherapy when ovarian recurrence occurs?}

\section{Surgery perspective}

I think the question is not whether surgery is superior to chemotherapy. Chemotherapy is clearly the standard therapy in patients with relapsed disease. We have already seen in the DESKTOP I trial [2] that platinum-based chemotherapy after surgery is an independent prognostic factor for further survival. The question is whether we could believe that surgery is an additional option comparable to primary treatment for patients in whom a platinum-based chemotherapy is planned. And now the answer is "Yes". Unfortunately, we cannot only speculate. Despite the positive result of the DESKTOP III trial regarding a significant improvement in median PFS from 14.0 to 19.6 months, we still have to wait for the data of the primary endpoint, OS. This wait (and believe or not) will have to be for two more years.

\footnotetext{
'Department of Gynecology and Gynecologic Oncology, Kliniken Essen-Mitte, Essen, Germany. ${ }^{2}$ Department of Gynecologic Oncology, European Institute of Oncology (IEO), Milan, Italy. ${ }^{3}$ Department of Gynecological-Obstetrical and Urological Sciences, "Sapienza" University of Rome, Rome, Italy. Correspondence to: Federica Tomao,

Department of Gynecological-Obstetrical and Urological Sciences, "Sapienza" University of Rome,

Viale del Policlinico 155, 00161 Roma, Italy.

Phone: +3906 4940550 - Fax: +3906 49972564

E-mail: federica.tomao@uniroma1.it

CANCER BREAKING NEWS 2017;5(3):11-13

DOI: 10.19156/cbn.2017.0053
} 


\section{Medical oncology perspective}

I think that secondary surgery, when the disease is fully de-bulkable, might represent an integrative approach to be considered before a second line chemotherapy. Furthermore, it may be hypothesized that recurrent ovarian cancer, included platinum-sensitive disease, is characterized by a higher percentage of platinum-resistant clones. As a consequence, the resection of most of the tumor could potentially allow a better response to chemotherapy. However, the most important prognostic factor for PFS in secondary surgery is the achievement of complete resection as observed in the front-line approach. Thus, in my opinion, the crucial question about the surgical approach, besides patient selection, is the efficacy and the quality of cytoreductive surgery.

The DESKTOP III trial was designed with OS as the primary endpoint and, despite the fact that long-term follow-up data are still immature, a benefit in PFS has been observed in patients who underwent the surgical approach in selected centers. In conclusion, I strongly believe that de-bulking surgery should be considered, taking into account not only several patient- and tumor-related factors, but also the availability of highly specialized surgical expertise. For this reason, I think that inclusion criteria for secondary cytoreductive surgery should absolutely include the availability of a tertiary level referral center specialized in gynecologicaloncological surgery.

Thus, waiting for final analysis of OS, the use of secondary de-bulking surgery should be considered as an experimental approach, particularly in those centers that are not able to perform such highly specialized surgery.

\section{Do you believe that the AGO score is helpful in selecting those patients who might benefit from secondary cytoreductive surgery?}

\section{Surgery perspective}

Absolutely. The AGO score is definitely a useful tool for selecting patients in whom complete resection is feasible. However, we have to keep in mind that we developed and validated the AGO score only to provide an instrument for selecting patients with a high chance for complete resection, which could be included in a randomized trial to prove the efficacy of surgery at relapse. It does not make any sense to randomize all comers without selection for a surgical option. You need a certain rate of complete resection to prove the efficacy of surgery.

\section{Medical oncology perspective}

I believe that AGO score has been studied in complete agreement with those criteria that might influence the real benefit of a secondary surgical approach. However, I again stress that secondary surgery is not feasible in every case. When highly specialized surgeons are lacking, correct medical management should be preferred. However, new therapeutic approaches in second line medical treatment open new scenarios that may potentially modify the outcome of platinum-sensitive recurrent disease regardless of tumor resection. Moreover, according to the 2017 Gynecologic Cancer Intergroup (GCIG) consensus conference, the old categorization of recurrent disease on the basis of platinum-free interval will be probably abandoned. Thus, in the future, the selection criteria of patients who will benefit from secondary surgery is likely to be revised.

\section{Do you think that surgery beyond secondary can be suitable for multiple patients with recurrent ovarian cancer?}

\section{Surgery perspective}

Yes. There are also some publications showing that patients with complete resection at tertiary surgery also seem to benefit from the surgery. However, the rate of patients who are suitable for tertiary surgery is very low. Therefore, this is a very individual decision.

\section{Medical oncology perspective}

I think that surgery beyond secondary should be considered in very selected patients. For example, in those patients with lower chances of response to chemotherapy (i.e., low grade or mucinous tumors) or with high motivation to choose a surgical rather than a medical approach. By the way,

I observed that in DESKTOP III trial most of the patients were affected by G2-3 serous ovarian cancer. I think that a sub-analysis according to histotype should be considered.

\section{Which criteria can be helpful in selecting patients who might benefit from secondary cytoreductive surgery?}

\section{Surgery perspective}

There are multiple criteria we have to consider, even beyond the perhaps more obvious criteria like localization of relapse, platinum-free interval, and the AGO score. Further important factors are co-morbidities, course after the first surgery (major complications?), the extent of first surgery, histologic subtype (low grade?) - and the most important one is the wishes of the patient. 


\section{Medical oncology perspective}

Besides the AGO score criteria, other parameters should be considered: the availability of surgical teams able to achieve the complete resection of the tumor, as previously noted, the age of patients, their wishes and, last but not least, the accessible therapeutic options. Moreover, regardless of the survival benefit achievable with surgery, we must consider quality of life as one of our main outcomes; this aspect should be further investigated.

\section{References}

1. Du Bois A, Vergote I, Ferron G et al. Randomized controlled phase III study evaluating the impact of secondary cytoreductive surgery in recurrent ovarian cancer: AGO DESKTOP III/ENGOT ov20 [Abstract]. J Clin Oncol 2017;35(15_suppl):5501.

\section{Acknowledgments}

The Authors thank Ray Hill, an independent medical writer, who provided native English editing and journal styling on behalf of HPS.

\section{Conflicts of Interest}

The Authors declare there are no conflicts of interest in relation to this article.

2. Harter P, du Bois A, Hahmann M et al. Surgery in recurrent ovarian cancer: the Arbeitsgemeinschaft Gynaekologische Onkologie (AGO) DESKTOP OVAR trial. Ann Surg Oncol 2006;13(12):1702-10. 